\title{
Developing a device measuring the middle ear reflex in rat ears: A preliminary study
}

\author{
Jeong-Seo Kim \\ Department of Communication Sciences and Disorders, University of lowa, lowa City, IA, USA
}

Purpose: The middle ear muscle (MEM) reflex is the contraction of the stapedius and tensor tympani muscles in the middle ear cavity in response to loud sound, and relies on a healthy population of auditory nerve fibers. Thus, the MEM reflex can be used as a diagnostic test to quantify auditory nerve survival. This study aims to develop a device to measure the MEM reflex in an animal model, with a long-term goal to develop a biomarker for auditory nerve degeneration.

Methods: A source device was constructed using a hearing aid speaker attached to a long and thin tube to create high impedance. A probe consists of a microphone placed near the tip of the tube and the sound source. Sound pressure that reflects the MEM reflex was measured in the probe ear of anesthetized rats while an elicitor stimulus was presented in an opposite ear.

Results: The probe to measure the MEM reflex was verified having a constant volume velocity sensitive to changes in sound pressure. Increases in amplitude of sound pressure were apparent when the stimulation signal was presented in the stimulating ear and the amplitude was decreased to baseline when the signal was turned off. After sacrifice of the animal, the reflex was absent.

Conclusions: Results showed the feasibility of the probe with high source impedance to measure the MEM reflex in animal ears. Absent reflex after animal sacrifice indicates that the MEM reflex measured using this probe was not an artifact or biological noise.

Keywords: Middle ear muscle reflex, Impedance, Rat

\section{INTRODUCTION}

Auditory function declines with aging and noise exposure over years. Damaged cochlear structures due to noise exposure or aging may lead to the degeneration of auditory nerve fibers $[1,2]$. Listeners can experience greater difficulty in understanding in adverse listening environments with background noise or high reverberation. These individuals can experience degraded temporal resolution even when audiometric thresholds are within normal range [3,4]. Well-preserved audiometric thresholds may indicate an absence of peripheral pathology in these individuals, however, this is less informative when the dominant pathology is not sensory.

Auditory nerve fibers are primary sensory neurons connecting hair cells to auditory brainstem. It has been known that the loss of auditory nerve fibers may be contributor to functional auditory decline $[2,5]$. Recent evidences in animal studies indicate that even temporary damage to the cochlea, followed by recovery, can cause auditory nerve

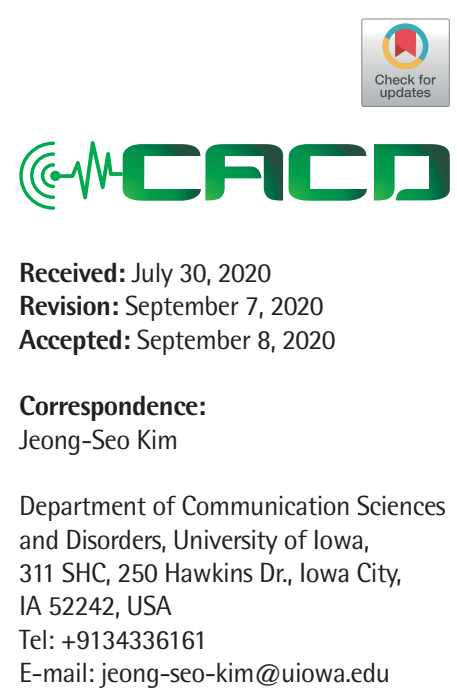

(C) 2020 The Korean Association of SpeechLanguage Pathologists

This is an Open Access article distributed under the terms of the Creative Commons Attribution NonCommercial License (https://creativecommons.org/ licenses/by-nc/4.0/) which permits unrestricted noncommercial use, distribution, and reproduction in any medium, provided the original work is properly cited. 
degeneration and progress with aging [3]. Guinea pigs were exposed to loud octave band noise (4-6 kHz) for two hours and retested for cochlear function at varying post exposure times. After noise exposure, a significant loss of presynaptic ribbons throughout the basal half of the cochlea was observed. Temporary threshold shifts after noise exposure were great both in the auditory brainstem response (ABR) and distortion product otoacoustic emissions (DPOAEs) as well as behavioral thresholds. Although thresholds were completely recovered after ten days of exposure, delayed and progressive loss of cochlear neurons over many months occurred. Two years after noise exposure, considerable loss of spiral ganglion cells in the basal turn in acoustic injury group was apparent compared to age-matched controls. In another animal study, cochlear responses were measured in mice at ages from four weeks to the end of their life span (144 weeks) in order to determine an effect of aging on anatomical damage [4]. Inner hair cell synaptic decline and spiral ganglion cell loss began at weaning ( 4 weeks) and steadily declined until death. Spiral ganglion cell counts reached $40 \%$ in old mice at 144 weeks indicating auditory nerve degeneration progressed with aging.

Neurophysiology studies suggest that noise-induced neuropathy is likely to be selective for low spontaneous rate nerve fibers [6]. There are at least two different populations of auditory nerve fibers, identified by their spontaneous discharge rate [7]. Low spontaneous rate fibers consist approximately $40 \%$ of neural population and have high thresholds and wider dynamic ranges than their higher spontaneous rate counterparts. Low spontaneous rate fibers are used to code for high signal levels while high spontaneous rate fibers are used to code for lower signal levels. Low spontaneous rate fibers are less susceptible to masking in the presence of continuous broadband noise than high spontaneous fibers [8]. Therefore, it is believed that low spontaneous rate fibers are important contributor for listening in background noise. Unfortunately, damage due to noise exposure and aging may cause degeneration of the low spontaneous rate fibers. Listening with the background noise can be altered while hearing thresholds remain normal. This may be related to the cause of 'hidden hearing loss' which is one of common complaints from individuals with hearing loss. People with hidden hearing loss has normal hearing sensitivity with difficulty in noisy environment [9]. They may have neural degeneration caused by noise exposure and/or aging without loss of cochlear hair cells.

Irreversible neural degeneration is a common result of acoustic overstimulation and aging, even though threshold sensitivity is not altered. A problem with current diagnostic hearing test procedures is that they categorize hearing disorders as "sensorineural" and do not distinguish the site of lesion within the cochlea or discriminate cochlear and neural disfunction. Standard hearing tests are not sensitive to characterize auditory nerve degeneration. Behavioral thresholds are not affected by neuronal damage when hair cells are still functioning as noted in animal studies [3,4]. Current hearing tests only provide rough estimation of underlying anatomical pathology [10].

New diagnostic test battery is needed to identify and monitor anatomic changes in the inner ear and central auditory system resulting hearing loss and the physiology of surviving anatomical structures. In that sense, developing a biomarker for auditory nerve degeneration may help differential diagnosis for at risk group and provide appropriate counseling by predicting outcomes with hearing aid or cochlear implant according to an etiology of hearing loss. Among current diagnostic tools, an amplitude of ABR wave I that reflects summed activity of auditory nerve fibers contacting inner hair cells may provide information about the functional integrity of auditory nerve fibers $[4,6]$. ABR wave I amplitude of the noiseexposed animals was significantly reduced compared to the control group, despite complete recovery of ABR thresholds after two weeks post exposure [6]. In order to use the ABR wave I as a biomarker in humans, more researches are needed to develop a reliable protocol to measure auditory nerve degeneration with less inter-subject variability.

Another candidate for a biomarker of auditory nerve degeneration is measuring the middle ear muscle (MEM) reflex. This acoustic middle ear reflex is an involuntary muscle contraction in the middle ear in response to high-intensity sound stimuli. This usually occurs at $85 \mathrm{~dB}$ HL in normal hearing individuals that may offer some minimal protection against noise-induced hearing loss. The MEM reflex is regarded as a robust measure of the integrity of auditory nerve pathways and sensitive to alterations of the auditory nerve. The MEM reflex has been utilized as a routine diagnostic tool in the audiology clinic to identify an ear with retrocochlear pathology. The acoustic reflex (AR) test has a long history of clinical use in defining middle ear, cochlear, and the seventh (facial) and eighth (vestibulocochlear) cranial nerve disorders [11]. In cases with retrocochlear site of lesion, the acoustic reflex threshold (ART) is usually elevated or absent and the reflex amplitude is decayed during continuous stimulation $[12,13]$. Elevated or absent thresholds and decay in the amplitude of 
the reflex in combination have high hit rate of $84 \%$ and particularly useful to identify small acoustic tumors located near the eighth cranial nerve [13]. In addition, the MEM reflex was absent or significantly reduced in individuals with auditory neuropathy, noise-induced tinnitus with normal or near normal audiometric thresholds who are suspected to have 'hidden hearing loss' [14-16]. These findings indicate the clinical potential of the MEM reflex as a sensitive noninvasive diagnostic test for characterizing the status of the auditory nerve.

Determining the feasibility of the MEM reflex as a diagnostic test to identify auditory nerve degeneration can be accomplished by recording the MEM reflex in animal models before and after application of a drug to cause damage to auditory nerve fibers. Since the MEM reflex is routinely used in the audiology clinic, translation from animal to human as a biomarker for auditory nerve survival would be transparent. Using commercially available acoustic immittance bridge constructed based on human ear canal measurements of acoustic impedance and admittance, and the related quantities of acoustic reflectance and power absorbance may not be optimal to measure the MEM reflex on animal ears. Impedance measurements made in the ear canal relate to the impedance at the eardrum that is significantly influenced by the dimensions and shape of the ear canal and the stiffness and density of the ear canal wall [17]. The size of ear canal and middle ear cavity, the hearing acuity, the reflex sensitivity, and the middle ear transmission change by the reflex in animal ears have shown different characteristics compared to humans [18-21]. For example, the best frequency to elicit the acoustic reflex lies between $2-8 \mathrm{kHz}$ in rabbits and $3 \mathrm{kHz}$ in rat ears, while those for human ears are between 1 and $2 \mathrm{kHz}$ [21]. ART measurements using commercially available diagnostic bridges are usually conducted at 500, 1,000, 2,000, and 4,000 Hz, however, results at $4,000 \mathrm{~Hz}$ are less reliable in many normal hearing young adults with elevated thresholds [22]. Testing high frequencies on animal ears by using this commercial tympanometer may not be optimal to measure the MEM reflex accurately. In addition, the MEM reflex of the most animal ears consists of two striated muscles (the stapedius and tensor tympani) responded in tandem. In contrast to animals in which both muscles are involved in the reflex to loud sounds, the dominant MEM reflex in human ears originates from the stapedius muscle [23]. Considering these anatomical and physiological differences between animals and humans, developing a sensitive tool that reflects changes in the MEM reflex in animal models should be proceeded in order to deter- mine the clinical value of this diagnostic technique to estimate neural survival.

This study aims to develop a device to measure the MEM reflex and test the feasibility of this tool in an animal model. The probe is constructed using device parameters suitable for animal ears to enhance the reliability of measurements. The hypothesis is that the amplitude of sound pressure measured using this custom probe will be noticeably increased when the signal is given in the stimulation ear which reflects the presence of the MEM reflex. If successful, this device can be used to measure the MEM reflex reliably in animal ears and further applied as a diagnostic technique to quantify the population of present auditory nerve fibers in humans as a longterm goal.

\section{METHODS}

\section{Subjects}

Three adult Long Evans rats weighing between 100-200 grams were used as subjects. Only animals with normal hearing estimated by a positive startle reflex were included in this study. All experimental procedures were approved by the Institutional Animal Care and Use Committee of the University of Kansas Medical Center and were carried out in accordance with the National Institutes for Health (NIH) guide for the Care and Use of Laboratory Animals.

Historically, rabbit was used to study the MEM reflex, however, more recent studies have been implemented on rats with improvements in anatomic and pharmacologic techniques to identify MEM reflex pathways [21,24]. In addition, this animal model was chosen because the surgical approach to the middle ear muscles is relatively straightforward.

\section{Equipment}

\section{Theory}

Impedance is related to sound pressure by $\mathrm{P}=\mathrm{ZU}$, where $\mathrm{P}$ is sound pressure in Pascals, $\mathrm{Z}$ is acoustic impedance in Ohms, and $\mathrm{U}$ is volume velocity in $\mathrm{m}^{3} / \mathrm{sec}$. This formula describes the relationship between impedance and sound pressure. The MEM reflex can be measured by changes in the impedance of the middle ear. When stapedius and tensor tympani muscles contract with presence of acoustic stimulation, impedance $(\mathrm{Z})$ of the middle ear changes and therefore sound pressure $(\mathrm{P})$ changes if volume velocity $(\mathrm{U})$ remains constant. The desirable condition is to have a speaker delivering a probe signal to the testing ear at a constant volume velocity inde- 
pendent of the load impedance coupled to the source. In this experiment, the load impedance $\left(Z_{e c}\right)$ is the impedance at the entrance of rat ear canal. Changes in $Z_{\text {ec }}$ produce proportional changes in sound pressure (P), thus, changes in $\mathrm{Z}$ are reflected in changes in the sound pressure of the probe signal. An ideal probe should selectively measure changes in sound pressure that reflects changes in impedance of the middle ear.

A probe consists of a speaker that generates volume velocity and a microphone that measures resulting sound pressure changes in the middle ear. The impedance of the probe placed in an ear canal split into the source impedance $\left(Z_{s}\right)$ and load impedance $\left(Z_{e c}\right)$. A probe delivering sound volume velocity $\left(U_{s}\right)$ is considered as the source and related impedance is designated as the source impedance $\left(Z_{s}\right)$. Once the probe placed into the ear canal, the volume of air in the canal, middle ear and inner ear structures together constitute the load impedance $\left(Z_{e c}\right)$. Combination of the source and load impedances can be modeled by Norton equivalent circuit (Figure 1). Sound pressure in the ear canal $\left(P_{e c}\right)$ is related to impedance and volume velocity by $P_{e c}=\frac{U_{s} Z_{s} Z_{e c}}{Z_{s}+Z_{e c}}=\frac{U_{s} Z_{s} Z_{e c}}{1+\frac{Z_{e c}}{Z_{s}}}$.

This formula shows that sound pressure in the ear canal $\left(P_{e c}\right)$ is influenced by the impedance of the speaker, tube, and microphone $\left(Z_{s}\right)$. If $Z_{s}$ is too small, an ability to quantify changes in sound pressure may limit because of the instrumental noise and sensitivity of the microphone. Allowing the source impedance goes infinite $\left(Z_{s}>>>Z_{e c}\right)$ is ideal to make a probe highly sensitive to changes in sound pressure.

\section{Developing a probe}

In order to create a probe with high source impedance $\left(Z_{s}\right)$, a hearing aid speaker was attached to a long and thin tube with small diameter. Ravicz et al. [25] showed that magnitude of the load impedance $\left(Z_{e c}\right)$ for rodent with the bulla open was approximately 1,000 CGS Ohms at $800 \mathrm{~Hz}$.

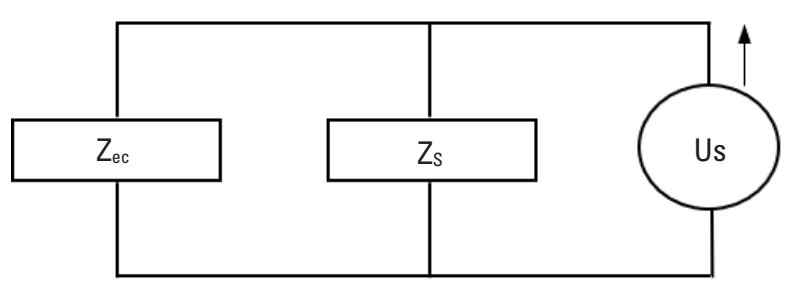

Figure 1. Norton equivalent circuit model that describes the acoustic environment to measure the MEM reflex in an animal model. Us indicates the acoustic volume velocity while $Z_{s}$ represents the source impedance and $Z_{e c}$ is the input impedance loaded into the ear canal.
Using a mathematical model of a cylinder regarding viscothermal properties [26], a size of the tube was determined to have a length of $10 \mathrm{~cm}$ with $0.7 \mathrm{~mm}$ diameter.

This was able to provide a ten times higher source impedance (10778 CGS Ohms) than $Z_{e c}$ reported by Ravicz et al. [25]. After the size of a tube was determined, a hearing aid speaker attached to an end of the tube. The tube coupled with a speaker and a microphone to measure sound pressure assemble the probe as illustrated in Figure 2.

\section{Procedures}

Rats were sedated with ketamine and xylazine $(0.1 \mathrm{~mL} / 100$ gram). Animals were secured on a head holder and wrapped with a heating blanket (Harvard Apparatus, Holliston, MA, United States) to maintain body temperatures at 37 degrees Celsius. The MEM reflex was measured via the probe assembly placed on the probe ear while loud signal was delivered to an opposite ear (stimulating ear) to evoke contralateral MEM reflex (Figure 3). Broadband noise was presented at $104 \mathrm{~dB}$ SPL via an ER-2 headphone (Etymotic Research, Elk Grove Village, IL, United States) attached to a speculum and sealed into an ear canal of the stimulating ear. Sound pressure was monitored with a microphone (ER7C, Etymotic Research) to determine signal levels. In the probe ear, the probe was positioned into the ear canal and $800 \mathrm{~Hz}$ probe tone was delivered at low to moderate level. When contralateral MEM reflex was activated by presence of high level noise in the stimulating ear, sound level changes of $800 \mathrm{~Hz}$ probe tone was recorded

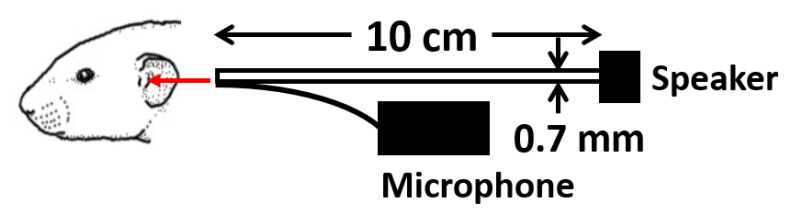

Figure 2. Dimensions of the probe assembly positioned to an animal ear.

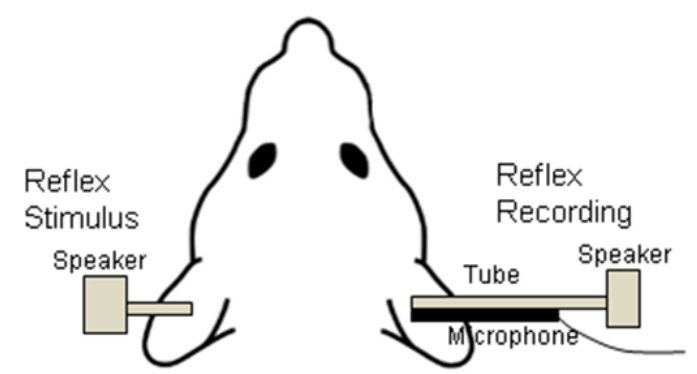

Figure 3. Schematic illustration of the experimental set up to measure the MEM reflex in rat ears. 
via a microphone placed in the probe ear. The probe stimulus ( $800 \mathrm{~Hz}$ low tone) was recorded for three seconds followed by simultaneous presentation of the probe and stimulation tone (broadband noise) for four seconds. Subsequently, the probe tone alone was recorded for three seconds.

\section{RESULTS}

\section{Verification of a probe}

In order to measure the MEM reflex reliably in animal ears, the probe should have constant volume velocity (U) over a wide range of impedances. The probe constructed for this experiment was verified by comparing changes in sound pressure (P) and impedance (Z) while varying cavity volumes. The probe was sealed into a $1 \mathrm{cc}$ syringe with clay for verification (Figure 4). Sound pressure at $800 \mathrm{~Hz}$ was measured via a microphone (ER7C, Etymotic Research) at seven different volumes $(0.3-0.15 \mathrm{cc})$ adjusted by a stopper. Impedance of the syringe was calculated using a mathematical model of a cylinder considering viscothermal characteristics of the probe (Benade) [26]. Sound pressures measured at varied cavity volumes and corresponding impedances were correlated and linear regression line was plotted. Figure 5 showed a proportional relationship between sound pressure and impedance with high correlation coefficient $(r=0.9922)$. Changes in sound pressure reflect changes in impedance over an expected range of impedance in a rat ear. Considering the formula $(\mathrm{P}=\mathrm{ZU})$, this suggests that the probe used in this study was able to maintain constant volume velocity (U).

\section{Measuring the MEM reflex}

The MEM reflex of animal ears can be detected by measuring sound pressure in the probe ear. Figure 6 illustrates changes in sound pressure measured over the time course. The probe stimulus (800 Hz low tone) was presented for first three seconds and followed by simultaneous presentation of the probe and stimulation signal (broadband noise at $104 \mathrm{~dB}$ SPL) for

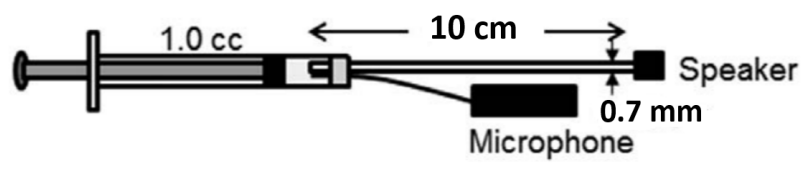

Figure 4. Illustration of the probe assembly used for verification. A tube coupled with a hearing aid speaker and a microphone were inserted into a 1 cc syringe with clay. Sound pressure was measured via a microphone while the volume of the syringe was varied by a stopper. next four seconds. Then, the probe tone alone was presented for the last three seconds.

Changes in sound pressure was determined by difference in amplitudes where the stimulating signal was turned on and off. Acoustic pressure remained relatively stable until high level noise signal was presented in the stimulating ear and this was considered as baseline. As noted in an envelope of

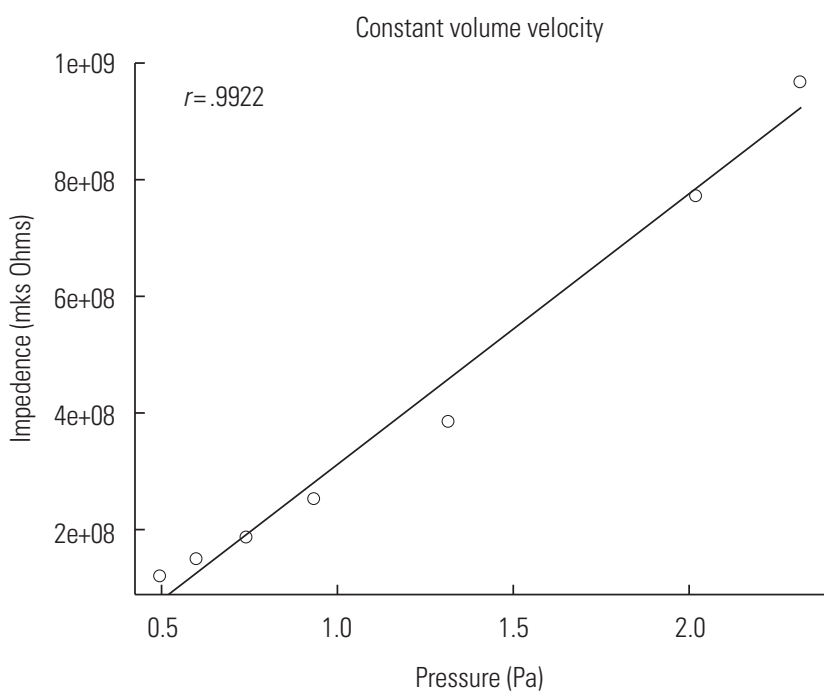

Figure 5. Correlation between sound pressure measured at different cavity volumes of the syringe (abscissa) and corresponding impedance calculated by a mathematical model of a cylinder (ordinate). Diagonal line indicates a linear regression line fitted with a correlation coefficient $(r=.9922)$.

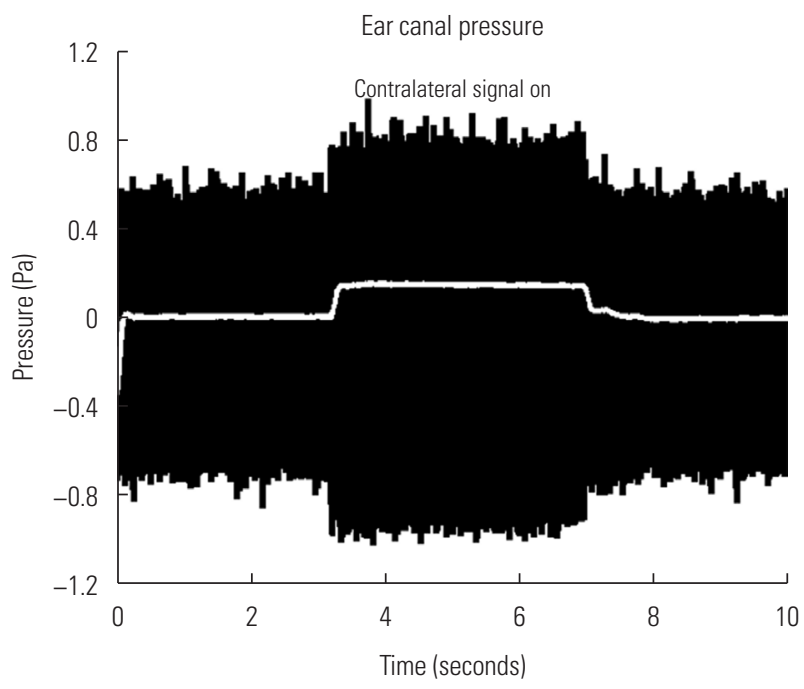

Figure 6. Sound pressure changes measured over the time course. The probe tone ( $800 \mathrm{~Hz}$ low tone) was only provided at the first and last three seconds, while high level broadband noise was given in the stimulating ear ('Contralateral signal on') with the probe tone for four seconds in the middle. Change in sound pressure after rectification and low-pass filtering is shown in white line, while raw magnitudes are shown in black. 


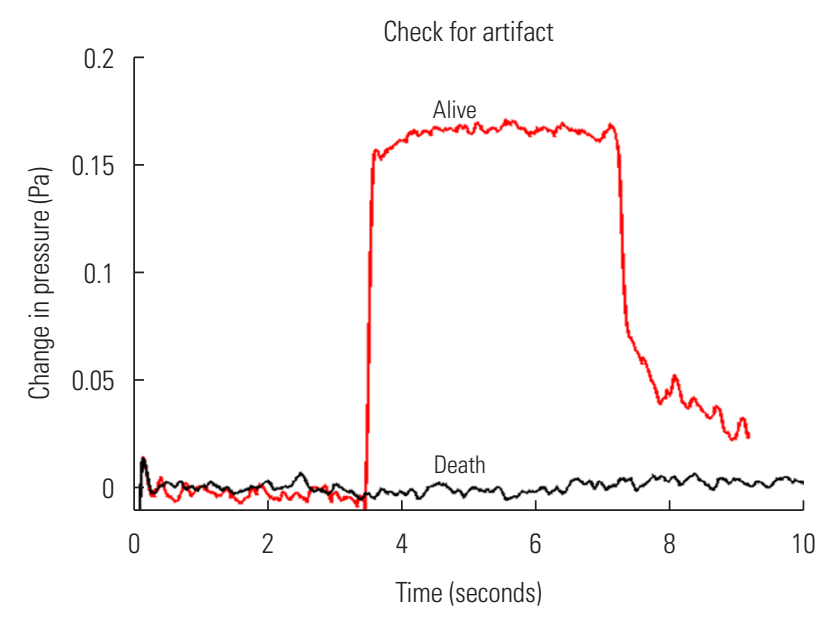

Figure 7. Checking for instrumentation and biologic artifact. Sound pressure was measured before and after animal sacrifice. Changes in sound pressure (a trace named 'Death') were absent when an animal was sacrificed compared to when an animal is alive (a trace named 'Alive'), indicating the response was not due to instrumentation noise or acoustic artifact.

black traces in Figure 6, increasing pattern in sound pressure with presence of the stimulation signal ('contralateral signal on') was apparent. Then, the amplitude of sound pressure was decreased to baseline when the signal was absent. Raw measurements (black traces, Figure 6) were rectified and lowpass filtered to quantify sound pressure changes over time (white line in the middle, Figure 6). While the high level signal was presenting, sound pressure was increased by 0.2 pascal on average. This trend was visually detectable via an oscilloscope and repeatable in tested animal ears.

\section{Checking for an artifact}

Instrumental and biologic artifact or noise was also checked in order to verify the reliability of measured impedances. Figure 7 shows sound pressure measured in a single animal before and after sacrifice. Same broadband noise at $104 \mathrm{~dB}$ SPL was presented to the stimulating ear and sound pressure was measured in the probe ear while an animal was sedated and an hour after sacrifice. When an animal was alive, sound pressure changed approximately 0.15 pascal. After sacrifice, changes in response was absent. This indicates that the measured response was truly the MEM reflex, not from an instrumental artifact or any noise interference.

\section{DISCUSSION}

In this preliminary study, the probe was created with high source impedance and constant volume velocity and its feasi- bility was tested to measure the MEM reflex in rat ears. We hypothesized that changes in amplitude of sound pressure measured using this probe will be noticeable when the stimulation signal was presented in the stimulating ear. As expected, the reflex amplitude measured using this probe was considerably increased when the broadband noise was on and decreased to the baseline level when the noise was off. An increase in the amplitude of sound pressure indicates a larger contraction of the stapedius and/or tensor tympani muscle in an animal ear. This implies that the custom probe used in this experiment was sensitive to reflect the activity of middle ear muscle reflex elicited by the presentation of loud stimulus in the stimulating ear.

The MEM reflex is sensitive to alteration of the auditory nerve that indicates potential as a biomarker for neural degeneration [12,13]. The MEM reflex is routinely tested in the audiology clinic to assist differential diagnosis for retrocochlear pathology such as a space-occupying tumor or mass impinging on the auditory nerve [11]. The acoustic reflex test for a clinical use measures changes in the signal level of a tone in the ear canal that as a result of contraction of the middle ear muscle and the decay in the magnitude of the reflex response. Mangham et al. [13] reported that normalized amplitude of the reflex response was the most sensitive parameter to identify an acoustic tumor in humans, compared to other parameters such as threshold, latency, rise and relaxation. The reflex amplitude was used as a parameter for the custom probe built for this study and changes in the amplitude were apparent when an elicitor signal was given. This indicates that the amplitude is a sensitive and appropriate parameter to characterize the presence of the MEM reflex in the animal model.

The MEM reflex has been commonly used in the clinic to identify the retrocochlear site of lesion. Recent studies suggested that the clinical application of the MEM reflex may be expanded to characterize subtle alterations in auditory nerve fibers. Auditory nerve fibers can be categorized into different groups depending on their spontaneous discharge rate such as high spontaneous rate fibers and low spontaneous rate fibers. Cochlear synaptopathy, also called 'hidden hearing loss', refers the damage toward synapses connecting low spontaneous rate fibers which are known to be vital for signal coding in noisy background $[3,27,28]$. Individuals with cochlear synaptopathy present difficulty hearing in noisy listening environment with intact hair cell population and normal audiograms. Considering this growing population with functional deficits in hearing without changes in hearing sensitivity, there have been efforts to adequately address their concerns and confirm 
with audiologic evaluations [29]. In recent studies, the MEM reflex was absent or significantly reduced in animals and humans with cochlear synaptopathy $[15,16]$. In mice with noiseinduced synaptopathy, the MEM reflex was greatly weakened with reduced low spontaneous rate fibers, despite normal auditory thresholds [15]. Similarly, the strength of the MEM reflex was significantly reduced in humans with noise-induced continuous tinnitus with normal or near normal audiometric thresholds who are suspected to have cochlear synaptopathy [16]. In human studies, wideband measure of the MEM reflex appeared to be more sensitive to changes of the strength of middle ear impedance that may be affected by cochlear synaptopathy than the standard clinical measure which uses a low frequency probe tone $(226 \mathrm{~Hz})$ in adults or a high frequency probe tone $(1,000 \mathrm{~Hz})$ in the neonatal population [3032]. These findings may suggest that the MEM reflex reflects subtle changes in auditory nerve fibers such as reduced low spontaneous rate fibers and can used as an objective tool to identify individuals at risk for damaged or degenerated auditory nerve induced by noise exposure, ototoxic drugs, and aging.

As a starting phase to determine if the MEM reflex can be used as a biomarker for auditory nerve degeneration, this preliminary study showed the feasibility of the custom probe to measure the MEM reflex in the animal model. If the reliability of this probe is ensured in a larger number of animal ears, this may increase the likelihood of success to apply this method before and after an application of a drug to cause neural degeneration in further studies and verify the clinical value of this diagnostic technique for translational purposes.

\section{ACKNOWLEDGMENTS}

This preliminary study was conducted as a Doctor of Audiology (Au.D.) capstone project of the department of hearing and speech in the University of Kansas Medical Center (Kansas City, KS, United States). This work was presented orally at the student research forum (March 2015) and the Au.D. research day (May 2015) of the University of Kansas Medical Center. The author thanks to Dr. Mark Chertoff and Dr. Marcelo Peppi for their help with the animal preparation and data analysis.

\section{REFERENCES}

1. Makary CA, Shin J, Kujawa SG, Liberman MC, Merchant SN. Agerelated primary cochlear neuronal degeneration in human tem- poral bones. J Assoc Res Otolaryngol. 2011;12:711-717.

2. Nadol JB. Patterns of neural degeneration in the human cochlea and auditory nerve: implications for cochlear implantation. Otolaryngology-Head and Neck Surgery. 2001;117:220-228.

3. Kujawa SG, Liberman MC. Adding insult to injury: cochlear nerve degeneration after "temporary" noise-induced hearing loss. J Neurosci. 2009;29:14077-14085.

4. Sergeyenko Y, Lall K, Liberman MC, Kujawa SG. Age-related cochlear synaptography: an early-onset contributor to auditory functional decline. J Neurosci. 2013;33:13686-13694.

5. Sugawara M., Corfas G., Liberman MC. Influence of supporting cells on neuronal degeneration after hair cell loss. J Assoc Res Otolaryngol. 2005;6:136-147.

6. Lin HW, Furman AC, Kujawa SG, Liberman MC. Primary neural degeneration in the guinea pig cochlea after reversible noise-induced threshold shift. JARO. 2011;12:605-616.

7. Furman AC, Kujawa SG, Liberman MC. Noise-induced cochlear neuropathy is selective for fibers with low spontanerous rates. J Neurophysiol. 2013;110:577-586.

8. Liberman MC, Epstein MJ, Cleveland SS, Wang H, Maison S. Toward a differential diagnosis of hidden hearing loss in humans. PLOS ONE. 2016;9:1-15.

9. Plack CJ, Barker D, Prendergast G. Perceptual consequences of "hidden" hearing loss. Trends Hear. 2014;18:1-11.

10. Humes LE, Tharpe AM, Bratt GW. Validity of hearing thresholds obtained from the rising portion of the audiogram in sensorineural hearing loss. J Speech Hear Res. 1984;27:206-211.

11. Musiek FE, Chermak GD. Psychophysical and behavioral peripheral and central auditory test. Handb Clin Neurol. 2015;129:313332.

12. Borg E. The intra-aural muscle reflex in retrocochlear pathology: a model study in the rabbit. Int J Audiol. 1977;16:316-330.

13. Mangham CA, Lindeman RC, Dawson WR. Stapedius reflex quantification in acoustic tumor patients. Laryngoscope. 1980;90: 242-250.

14. Berlin CI, Hood LJ, Morlet T, Wilensky D, St John P, Montgomery E, Thibodaux M. Absent or elevated middle ear muscle reflexes in the presence of normal otoacoustic emissions: a universal finding in 136 cases of auditory neuropathy/dis-synchrony. J Am Acad Audiol. 2005;16:546-553.

15. Valero ME, Hancock KE, Liberman CE. The middle ear muscle reflex in the diagnosis of cochlear neuropathy. Hear Res. 2016;332: 29-38.

16. Wojtczak M, Biem JA, Oxenham AJ. Weak middl-ear-muscle reflex in humans with noise-induced tinnitus and normal hearing may reflect cochlear synaptopathy. eNeuro. 2017;4(6): doi: 10.1523/ ENEURO.0363-17.

17. Rosowski JJ, Wilber LA. Acoustic immittance, absorbance, and reflectance in the human ear canal. Semin Hear. 2015;36:11-28.

18. Borg E. Acoustic middle ear reflexes: a sensory-control system. Acta Otolaryngol Suppl. 1972;304:1-34.

19. Moller AR. Acoustic reflex in man. J Acous Soc Am. 1962;34:15241534. 
20. Moller AR. The sensitivity of contraction of the tympanic muscles in man. Ann Otol Rhinol Laryngol. 1962;71:86-95.

21. Murata K, Ito S, Horikawa J, Minami S. The acoustic middle ear muscle reflex in albino rats. Hear Res. 1986;23:169-183.

22. Gelfand SA, Piper N. Acoustic reflex thresholds in young and elderly subjects with normal hearing. J Acous Soc Am. 1981;691: 295-297.

23. Borg E. On the neuronal organization of the acoustic middle ear reflex: a physiological and anatomical study. Brain Res. 1973;49: 101-123.

24. Relkin EM, Sterns A, Azeredo W, Prieve BA, Woods CI. Physiological mechanisms of onset adaptation and contralateral suppression of DPOAEs in the rat. J Assoc Res Otolaryngol. 2005;6:119135.

25. Ravicz ME, Rosowski JJ, Voigt HF. Sound-power collection by the auditory periphery of the Mongolian gerbil Meriones unguiculatus I: Middle-ear input impedance. J Acoust Soc Am. 1992;92:157177.

26. Benade AH. On the propagation of sound waves in a cylindrical conduit. J Acoust Soc Am. 1968;44:616-623.
27. Kujawa SG, Liberman MC. Synaptopathy in the noise-exposed and aging cochlea: primary neural degeneration in acquired sensorineural hearing loss. Hear Res. 2015;330:191-199.

28. Liberman MC, Kujawa SG. Cochlear synaptopathy in acquired sensorineural hearing loss: menifestations and mechanisms. Hear Res. 2017;349:138-147.

29. Barbee CM, James JA, Park JH, Smith EM, Johnson CE, Clifton S, Danhauer JL. Effectiveness of auditory measures for detecting hidden hearing loss and/or cochlear synaptopathy: a systematic review. Semin Hear. 2018;39:172-209.

30. Feeney MP, Keefe DH. Estimating the acoustic reflex threshold from wideband measures of reflectance, admittance, and power. Ear Hear. 2001;22:316-332.

31. Keefe DH, Feeney MP, Huner LL, Fitzpatrick DF. Aural acoustic stapedius-muscle reflex threshold procedures to test human infants and adults. J Assoc Res Otolaryngol. 2017;18:65-88.

32. Schrairer KS, Ellison JC, Fitzpatrick D, Keefe DH. Wideband ipsilateral measurements of middle-ear muscle reflex thresholds in children and adults. J Acoust Soc Am. 2007;121:3607-3616. 